\title{
Convergence Estimates for Essentially Positive Type Discrete Dirichlet Problems
}

\author{
By J. H. Bramble, B. E. Hubbard and Vidar Thomée
}

\begin{abstract}
In this paper we consider a class of difference approximations to the Dirichlet problem for second-order elliptic operators with smooth coefficients. The main result is that if the order of accuracy of the approximate problem is $\nu$, and $F$ (the right-hand side) and $f$ (the boundary values) both belong to $C^{\lambda}$ for $\lambda<\nu$, then the rate of convergence is $O\left(h^{\lambda}\right)$.
\end{abstract}

1. Introduction. Let $R$ with boundary $\partial R$ be a bounded domain in euclidean $N$-space $E^{N}$. We shall be concerned with the solution of the Dirichlet problem

$$
\begin{aligned}
L u & \equiv-\sum_{j, k=1}^{N} a_{j k}(x) \frac{\partial^{2} u}{\partial x_{j} \partial x_{k}}+\sum_{j=1}^{N} a_{j}(x) \frac{\partial u}{\partial x_{j}}+a_{0}(x) u=F \quad \text { in } R, \\
u & =f \text { on } \partial R,
\end{aligned}
$$

where $L$ is uniformly elliptic and $a_{0} \geqq 0$ in $R$. For the numerical solution it is common to cover $R$ by a square mesh with mesh-width $h$, and for "interior" meshpoints $x$ approximate the Eq. (1.1) by an equation of the form

$$
L_{h} u_{h}(x) \equiv h^{-2} \sum_{\beta} b_{\beta}(x, h) u_{h}(x+\beta h)=M_{h} F(x),
$$

where $\beta=\left(\beta_{1}, \cdots, \beta_{N}\right)$ has integer components and $L_{h}$ and $M_{h}$ are consistent with $L$ and the identity operator, respectively. For mesh-points near $\partial R$ one considers similarly equations of the form

$$
l_{h} u_{h}(x)=u_{h}(x)+\sum_{\beta \neq 0} b_{\beta}(x, h) u_{h}(x+\beta h)=m_{h}(F, f)
$$

which take into account both Eqs. (1.1) and (1.2). In much of the literature, it is assumed that $L_{h}$ is of positive type, or $\sum_{\beta} b_{\beta}(x, h) \geqq 0$ and $b_{\beta}(x, h) \leqq 0$ for $\beta \neq 0$ in (1.3), and this is the case that is considered in this paper. Similarly, the $b_{\beta}$ in (1.4) are often assumed $\leqq 0$; we shall assume here that

$$
\sum_{\beta}\left|b_{\beta}\right| \leqq \gamma<1
$$

and shall say then that the pair of operators $L_{h}$ and $l_{h}$ is of essentially positive type.

For many special schemes of the type described, convergence results are given in the literature. They are generally of the form that if the discrete problem (1.3), (1.4) approximates the continuous (1.1), (1.2) with order of accuracy $\nu$, then

$$
\left|u(x)-u_{h}(x)\right| \leqq C h^{\nu} .
$$

The constant $C$ here depends on the unknown solution $u$; in general one has had to assume that $u$, together with its derivatives of orders less than or equal to $\nu+2$, is bounded in $R$.

Received May 5, 1969. 
Thus in particular, if $N=2$ and

$$
L u \equiv-\Delta u \equiv-\frac{\partial^{2} u}{\partial x_{1}{ }^{2}}-\frac{\partial^{2} u}{\partial x_{2}{ }^{2}}
$$

a common approximation of (1.1) is the well-known "five-point" formula

$$
-\Delta_{h}{ }^{(5)} u(x)=h^{-2}\left\{4 u(x)-\sum_{|\beta|=1} u(x+\beta h)\right\}=F(x) .
$$

For this operator, and for the simplest possible boundary approximations, Gerschgorin [7] proved an estimate of the form (1.5) with $\nu=1$. Later Collatz [6] using linear interpolation near the boundary, improved the result to get (1.5) with $\nu=2$. Using instead of (1.6) the "nine-point" formula

$$
\begin{aligned}
-\Delta_{h}{ }^{(9)} u(x) & =\frac{1}{6 h^{2}}\left\{20 u(x)-4 \sum_{|\beta|=1} u(x+\beta h)-\sum_{\left|\beta_{1}\right|=\left|\beta_{2}\right|=1} u(x+\beta h)\right\} \\
& =F(x)+\frac{h^{2}}{12} \Delta_{h}{ }^{(5)} F(x),
\end{aligned}
$$

Bramble and Hubbard [3] showed that the operator $l_{h}$ in (1.4) can be chosen in such a manner that (1.5) holds for $\nu=4$. These authors [4] also constructed operators $L_{h}$ and $l_{h}$ in the case of a general $L(N=2)$ such that (1.5) holds with $\nu=2$.

It was observed by Bahvalov [1] in an important paper, seemingly not wellknown outside the Russian literature, that the regularity demands on the solution $u$ of the continuous problem in some cases can be relaxed by essentially two derivatives at the boundary without losing the convergence estimate (1.5) and that for still less regular $u$ one can obtain correspondingly weaker convergence estimates. Bahvalov used his error bounds to estimate the number of arithmetic operations needed to obtain $u$ to a prescribed accuracy. Related results were also obtained in special cases by Wasow [13], Laasonen [8], and by Volkov, cf. [11], [12], and references.

The purpose of this paper is to present a general theory which comprises all the special features mentioned. In doing so we shall express the estimates in terms of the data $F$ and $f$ of the problem rather than in terms of the unknown solution $u$; the main result will be of the type that if $F$ and $f$ both belong to $\mathrm{e}^{\lambda}$ for some $\lambda>\nu$, then an inequality of the form (1.5) holds. It will also be shown that if $F$ and $f$ are in $\mathfrak{C}^{\lambda}$ for $\lambda<\nu$, then error bounds of the form $O\left(h^{\lambda}\right)$ can be obtained. Since the effort is concentrated on the dependence of the regularity of $F$ and $f$, we shall assume that the coefficients and the boundary are infinitely differentiable.

The proofs will be based on new estimates for the discrete Green's function for the operator $L_{h}$. This estimate can be thought of as a discrete analogue of the estimate

$$
\int_{d(y)=\delta} G(x, y) d \delta \leqq C \delta, \quad x \in R
$$

for the continuous Green's function, where $d(y)$ is the distance from $y$ to $\partial R$. (In special cases such results were used by Volkov [12].) The transition between estimates in terms of the solution and the data $F$ and $f$ will be made by means of the 
Schauder estimates for second-order elliptic differential operators; at some points it will be convenient to use interpolation properties of Lipschitz spaces. These latter types of techniques also apply to other convergence problems in difference equations (cf. Peetre and Thomée [10] and Bramble, Kellogg, and Thomée [5]).

In a certain sense the results are not optimal as far as the regularity of $F$ is concerned; it will be shown in a forthcoming paper by Bramble [2] that the operator $M_{h}$ in (1.3) can be chosen in such a manner as to make it possible to further relax the regularity demands on $F$.

2. Preliminaries. We start by introducing some notation. For $\mathfrak{T} \subset E^{N}$, let $\mathcal{C}(\mathfrak{T})$ be the set of real-valued continuous functions on $\mathfrak{T}$ and define

$$
|u|_{\mathfrak{M}}=\sup _{x \in \mathfrak{M}}|u(x)| \text {. }
$$

In particular, if $\mathfrak{T}$ is a finite point-set, $\mathfrak{C}(\mathfrak{T})$ simply consists of all real-valued functions on $\mathfrak{T}$ and $|u|_{\mathfrak{M}}$ is always finite.

For a domain $R \subset E^{N}$ and $u \in \mathcal{C}(R), 0<\sigma \leqq 1$ we set

$$
H_{\sigma, \mathbb{R}}(u)=\sup _{x, x+y \in \mathscr{R} ; y \neq 0} \frac{|u(x+y)-u(x)|}{|y|^{\sigma}} .
$$

Let $\alpha=\left(\alpha_{1}, \cdots, \alpha_{N}\right)$ with $\alpha_{j}$ nonnegative integers and define $D^{\alpha} u=\left(\partial / \partial x_{1}\right)^{\alpha_{1}}$ $\cdots\left(\partial / \partial x_{N}\right)^{\alpha}{ }_{N}$. If $s$ is a positive real number and $s=S+\sigma$, where $S$ is an integer and $0<\sigma \leqq 1$, we say that $u \in \mathcal{C}^{s}(\bar{\Re})$ if $D^{\alpha} u \in \mathfrak{C}(\mathcal{R})$ for $|\alpha|=\sum_{j} \alpha_{j} \leqq S$ and if

$$
|u|_{s, R}=|u|_{R}+\sum_{|\alpha|=S} H_{\sigma, R}\left(D^{\alpha} u\right)
$$

is finite. We set $\mathfrak{C}^{x}(\bar{\Omega})=\bigcap_{s>0} \mathfrak{C}^{s}(\bar{\Omega})$.

For $u \in \mathfrak{C}(R)$ we also set

$$
Z_{\sigma, \mathbb{R}}(u)=\sup _{x, x \pm y \in \mathbb{R} ; y \neq 0} \frac{|u(x+y)-2 u(x)+u(x-y)|}{|y|^{\sigma}}
$$

and say again for $s=S+\sigma$, where $S$ is a nonnegative integer and $0<\sigma \leqq 1$, that $u \in \mathcal{C}_{z^{8}}(\bar{\Re})$ if $D^{\alpha} u \in \mathcal{C}(\Re)$ for $|\alpha| \leqq S$ and if

$$
|u|_{Z, s, \mathbb{R}}=|u|_{\mathbb{R}}+\sum_{|\boldsymbol{\alpha}|=S} Z_{\sigma, \mathbb{R}}\left(D^{\alpha} u\right)
$$

is finite. The finiteness of $H_{1, \Omega}(u)$ or $Z_{1, Q}(u)$ means that $u$ satisfies a Hölder condition or a Zygmund condition, respectively. Under the regularity assumptions below on $\partial \Re$ we have $\mathfrak{C}_{Z^{s}}(\bar{R})=\mathfrak{C}^{s}(\bar{R})$ for nonintegral $s$; for integral $s$ we have $\mathfrak{C}^{s}(\bar{R}) \subseteq$ $\mathfrak{C}_{Z}{ }^{s}(\bar{R})$.

Let $R_{\delta}=\{x ; x \in R ; d(x)>\delta\}$ where $d(x)$ is the distance from $x$ to the boundary $\partial R$ of $R$. We say that $u \in \mathfrak{C}^{s}(\mathcal{R})$ if $u \in \mathfrak{C}^{s}\left(\bar{\Omega}_{\delta}\right)$ for all $\delta>0$.

We shall always assume that $\partial R \in \mathcal{C}^{\infty}$, so that each point $x \in \partial R$ has a neighbor-

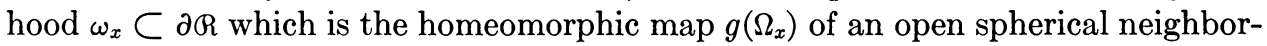
hood $\Omega_{x}$ of the origin in $E^{N-1}$ and $g_{j} \in \mathcal{C}^{\infty}\left(\bar{\Omega}_{x}\right), j=1, \cdots, N$ where $g=\left(g_{1}, \cdots, g_{N}\right)$. Since $R$ is a bounded domain we can by compactness cover $\partial R$ by a finite number of the sets $\omega_{x_{j}}$ so that $\partial R=\bigcup_{j=1}^{J} \omega_{x_{j}}$. Let $g^{(j)}$ be the mapping corresponding to $\omega_{x_{j}}$. We say that $f \in \mathcal{C}^{s}(\partial R)$ if $f\left(g^{(j)}\right) \in \mathfrak{C}^{s}\left(\bar{\Omega}_{x_{j}}\right)$ for $j=1, \cdots, J$, and define 


$$
|f|_{8, \partial R}=\max _{j}\left|f\left(g^{(j)}\right)\right|_{8, \Omega_{x_{j}}} \cdot
$$

The definition of $f \in \mathcal{C}_{Z^{8}}(\partial R)$ and $|f|_{z, s, \partial R}$ are analogous.

Consider now in the bounded domain $R$ the uniformly elliptic operator

$$
L u(x) \equiv-\sum_{j, k=1}^{N} a_{j k}(x) \frac{\partial^{2} u}{\partial x_{j} \partial x_{k}}+\sum_{j=1}^{N} a_{j}(x) \frac{\partial u}{\partial x_{j}}+a_{0}(x) u(x) \quad a_{j k}(x)=a_{k j}(x)
$$

so that for some constant $\epsilon_{0}>0$, real $\xi=\left(\xi_{1}, \cdots, \xi_{N}\right)$ and $x \in R$

$$
\sum_{j, k=1}^{N} a_{j k}(x) \xi_{j} \xi_{k} \geqq \epsilon_{0}|\xi|^{2} \quad|\xi|=\left(\sum_{j=1}^{N} \xi_{j}{ }^{2}\right)^{1 / 2} .
$$

We shall assume for simplicity that all coefficients are in $\mathfrak{C}^{\infty}\left(E^{N}\right)$ and also that $a_{0}(x) \geqq 0$.

Our aim is to discuss the approximate solution of the Dirichlet problem

$$
\begin{array}{rlll}
L u=F & \text { in } & \mathbb{R} \\
u=f & \text { on } & \partial R
\end{array}
$$

by finite difference methods.

We shall study finite difference approximations of $L$ of the form

$$
L_{h} u(x)=h^{-2} \sum_{\beta} b_{\beta}(x, h) u(x+\beta h)
$$

where $\beta=\left(\beta_{1}, \cdots, \beta_{N}\right)$ with integral components $\beta_{j}$. We assume that there are positive constants $h_{0}$ and $B$ such that $b_{\beta} \in \mathcal{C}^{\infty}\left(E^{N} \times\left[0, h_{0}\right]\right)$ and $b_{\beta}=0$ for $|\beta|>B$. We shall always assume that $L_{h}$ is consistent with $L$ so that for any $x$ and any $u$ sufficiently smooth

$$
\lim _{h \rightarrow 0} L_{h} u(x)=L u(x) .
$$

We shall further assume that $L_{h}$ is of positive type;i.e. for $h \leqq h_{0}$ and $x \in \mathbb{R}$ we have

$$
\begin{aligned}
\sum_{\beta} b_{\beta}(x, h) & \geqq 0, \\
b_{\beta}(x, h) & \leqq 0 \quad \beta \neq 0 .
\end{aligned}
$$

Let $E_{h}{ }^{N}$ be the set of mesh-points $x=\left(m_{1} h, \cdots, m_{N} h\right)$ where $m_{j}$ are integers. For $x \in E_{h}{ }^{N}$, the set $\left\{y ; y=x+\beta h, b_{\beta}(x, h) \neq 0\right.$ for $\left.h \leqq h_{0}\right\}$ is referred to as the set of neighbors of $x$; its convex hull in $E^{N}$ will be denoted by $\mathfrak{N}_{x}$. We set

$$
\begin{aligned}
\bar{R}_{h} & =\bar{\Re} \cap E_{h}{ }^{N} \\
R_{h} & =\left\{x ; x \in \bar{R}_{h}, \mathfrak{N}_{x} \subset \overline{\mathbb{R}}\right\} \\
\partial R_{h} & =\bar{R}_{h} \backslash R_{h} .
\end{aligned}
$$

The points in $R_{h}$ are called interior mesh-points; those of $\partial R_{h}$ are boundary meshpoints. We denote the set of real-valued functions defined on the above sets by $\bar{D}_{h}, \mathfrak{D}_{h}$, and $\partial D_{h}$, respectively.

In addition to the operator $L_{h}$ which will be used at interior mesh-points, we introduce an operator $l_{h}$ which will be related to the boundary values, 


$$
l_{h} u(x)=\sum_{\beta} b_{\beta}(x, h) u(x+\beta h) \quad x \in \partial R_{h} .
$$

We shall assume that $b_{0}(x, h) \equiv 1$ and that $b_{\beta}(x, h)=0$ for $|\beta|>B$ and for $x+$ $\beta h \notin \bar{R}_{h}$. No regularity will be assumed about the coefficients in $l_{h}$; instead we shall assume that there exists $\gamma<1$ such that

$$
\sum_{\beta \neq 0}\left|b_{\beta}(x, h)\right| \leqq \gamma, \quad x \in \partial R_{h}, h \leqq h_{0} .
$$

For the approximate solution of the Dirichlet problem (2.1), (2.2) we now consider a discrete problem

$$
\begin{aligned}
L_{h} u_{h}=M_{h} F & \text { on } R_{h} \\
l_{h} u_{h}=m_{h} \mathcal{F} & \text { on } \partial R_{h} .
\end{aligned}
$$

Here $M_{h}$ is a bounded linear operator from $\mathfrak{C}(\bar{R})$ into $D_{h}, \mathcal{F}=(F, f) \in \mathfrak{C}(\bar{R}) \times$ $\mathcal{C}(\partial R)$ and $m_{h}$ is a bounded linear operator from $\mathfrak{C}(\overline{\mathcal{R}}) \times \mathfrak{C}(\partial \Re)$ into $\partial D_{h}$. We shall prove later (Lemma 5.3) that this problem has a unique solution for small $h$, and our aim is to study the convergence of this solution $u_{h}$ to the solution of (2.1), (2.2).

We say that the discrete problem approximates the continuous problem with order of accuracy $\nu$ if for any $\lambda, \mu$ with $0 \leqq \lambda \leqq \nu, 0 \leqq \mu \leqq \nu$ there is a constant $C$ such that

$$
\begin{aligned}
\left|L_{h} u(x)-M_{h} L u(x)\right| & \leqq C h^{\lambda}|u|_{2+\lambda, \Re_{x}}, \quad u \in \mathbb{e}^{2+\lambda}\left(\bar{\Re}_{x}\right), \quad x \in R_{h}, \\
\left|l_{h} u-m_{h}(L u, \tilde{u})\right|_{\partial R_{h}} & \leqq C h^{\mu}\left(|u|_{\mu, \Omega}+|L u|_{\Re}\right), \quad u \in \mathbb{C}^{\mu}(\bar{\Omega})
\end{aligned}
$$

where $\tilde{u}$ denotes the restriction of $u \in \mathcal{C}(\bar{R})$ to $\partial R$. By the consistency between $L_{h}$ and $L, M_{h}$ is then an approximation of the identity operator.

We can now state our main result:

Theorem. Assume that the operators $L, L_{h}, l_{h}, M_{h}$, and $m_{h}$ satisfy the above assumptions and that the discrete problem (2.5), (2.6) approximates the Dirichlet problem (2.1), (2.2) with order of accuracy $\nu$. Let $u_{h}$ and $u$ be the solutions of the discrete and continuous problems, respectively. Then for $\lambda, \mu \geqq 0, \lambda, \mu \neq \nu$, there is a constant $C$ such that for $F \in \mathfrak{e}_{Z^{\lambda}}(\bar{\Re}), f \in \mathfrak{C}_{Z^{\mu}}(\partial R)$ we have

$$
\left|u-u_{h}\right|_{R h} \leqq C\left\{h^{\min (\lambda, \nu)}|F|_{Z, \lambda, \Re}+h^{\min (\mu, \nu)}|f|_{Z, \mu, \partial R}\right\} .
$$

Further, if $F \in \mathcal{C}^{\lambda}(\bar{\Re})$ for some $\lambda>0$, and $f \in \mathcal{C}(\partial R)$, we have

$$
\lim _{h \rightarrow 0}\left|u-u_{h}\right|_{R_{h}}=0 \text {. }
$$

The proof of this result will be given in Section 5 .

3. Positive Type Operators and Green's Functions. Throughout this section we shall assume that $L, L_{h}$, and $R$ satisfy the assumptions of Section 2 . We start with a lemma concerning the structure of positive type operators.

LEMmA 3.1. There are positive constants $h_{0}$ and $\mathfrak{K}$ such that for any $x \in R_{h}$ and any $\eta \in E^{N}$ with $|\eta|=1$ there is a $\beta \in E^{N}$ with integral components such that for $h \leqq h_{0}$

$$
\begin{gathered}
\text { (i) }(\beta, \eta) \geqq \mathcal{K}, \\
\text { (ii) }-b_{\beta}(x, h) \geqq \mathcal{K}, \\
\text { where }(\beta, \eta)=\sum_{j=1}^{N} \beta_{j} \eta_{j} .
\end{gathered}
$$


Proof. By Taylor's theorem we have for smooth $u$,

$$
\begin{aligned}
L_{h} u(x)= & u(x) h^{-2} \sum_{\beta} b_{\beta}(x, h)+\sum_{j} \frac{\partial u}{\partial x_{j}} h^{-1} \sum_{\beta} \beta_{j} b_{\beta}(x, h) \\
& +\frac{1}{2} \sum_{j, k} \frac{\partial^{2} u}{\partial x_{j} \partial x_{k}} \sum_{\beta} \beta_{j} \beta_{k} b_{\beta}(x, h)+o(1) \quad \text { as } h \rightarrow 0
\end{aligned}
$$

and so from the consistency we conclude that

$$
\begin{aligned}
\sum_{\beta} b_{\beta}(x, h) & =h^{2} a_{0}(x)+o\left(h^{2}\right) \quad \text { when } h \rightarrow 0, \\
\sum_{\beta} \beta_{j} b_{\beta}(x, h) & =h a_{j}(x)+o(h) \quad \text { when } h \rightarrow 0, \\
\sum_{\beta} \beta_{j} \beta_{k} b_{\beta}(x, h) & =-2 a_{j k}(x)+o(1) \quad \text { when } h \rightarrow 0 .
\end{aligned}
$$

In particular, if $|\eta|=1$, we obtain after multiplication of (3.1) by $\eta_{j} \eta_{k}$ and summation over $j$ and $k$, and using the ellipticity of $L$,

$$
\begin{aligned}
-\sum_{\beta}(\beta, \eta)^{2} b_{\beta}(x, h) & =2 \sum_{j, k} a_{j k}(x) \eta_{j} \eta_{k}+o(1) \quad \text { when } h \rightarrow 0, \\
& \geqq 2 \epsilon_{0}+o(1) \quad \text { when } h \rightarrow 0
\end{aligned}
$$

for sufficiently small $h$, uniformly in $x$ and $\eta$. Similarly,

$$
\sum_{\beta}(\beta, \eta) b_{\beta}(x, h)=o(1) \quad \text { when } h \rightarrow 0 .
$$

Since $\beta$ and $b_{\beta}$ are uniformly bounded, to prove the statement it is clearly sufficient to prove

$$
\inf _{h \leqq h_{0} ; x \in R_{h}}\left[-\sum_{(\beta, \eta)>0}(\beta, \eta) b_{\beta}(x, h)\right]>0 .
$$

But by (3.2) and (3.3) we have for some positive $h_{0}$ that for $h \leqq h_{0}, x \in R_{h}$, and $|\eta|=1$,

$$
\begin{aligned}
-\sum_{(\beta, \eta)>0}(\beta, \eta) b_{\beta}(x, h) & =-\frac{1}{2} \sum_{\beta}|(\beta, \eta)| b_{\beta}(x, h)+o(1) \\
& \geqq-(2 B)^{-1} \sum_{\beta}(\beta, \eta)^{2} b_{\beta}(x, h)+o(1) \geqq B^{-1} \epsilon_{0}
\end{aligned}
$$

which thus proves the lemma.

The above lemma tells us that given any $x \in R_{h}$ and any plane through $x$, there is a neighbor of $x$ on each side of the plane with distance greater than or equal to $\mathcal{K} h$ from the plane and corresponding to a coefficient with $\left|b_{\beta}(x, h)\right| \geqq \mathcal{K}$.

We can now prove the following maximum principle:

Lemma 3.2. Let $h \leqq h_{0}$ where $h_{0}$ is the constant in Lemma 3.1. Then if $v \in \bar{D}_{h}$ satisfies $L_{h} v \geqq 0$ on $R_{h}, v \geqq 0$ on $\partial R_{h}$, we have $v \geqq 0$ on $\bar{R}_{h}$.

Proof. Assume the conclusion is false, that $v$ has a negative minimum $v\left(x^{(0)}\right)$ on $R_{h}$. Since $L_{h}$ is of positive type we have

$$
v\left(x^{(0)}\right) \geqq \sum_{\beta}\left(-b_{0}(x, h)^{-1} b_{\beta}(x, h)\right) v\left(x^{(0)}+\beta h\right)
$$

and since the coefficients on the right are nonnegative and have sum at most 1 , we 
conclude that for all neighbors corresponding to nonzero $b_{\beta}, v\left(x^{(0)}+\beta h\right) \leqq v\left(x^{(0)}\right)$. Using Lemma 3.1 with $\eta=e_{1}$, we find that for one such neighbor $x^{(1)}, x_{1}{ }^{(1)} \geqq x_{1}{ }^{(0)}+$ $\mathcal{K} h$. Iterating this argument we find a sequence of points $x^{(j)}, j=1,2, \cdots$, such that

$$
v\left(x^{(j)}\right) \leqq v\left(x^{(0)}\right) \quad x_{1}{ }^{(j)} \geqq j h \mathcal{K}+x_{1}{ }^{(0)} .
$$

But by the boundedness of $R$, after a finite number of steps, $x^{(j)} \in \partial R_{h}$, and thus $v\left(x^{(j)}\right) \leqq 0$, which is a contradiction.

We can now conclude:

Lemma 3.3. The discrete problem

$$
\begin{aligned}
L_{h} v & =F & & \text { on } R_{h} \\
v & =f & & \text { on } \partial R_{h}
\end{aligned}
$$

has a unique solution $v \in \bar{D}_{h}$ for any $F \in \bar{D}_{h}$ and $f \in \partial D_{h}$.

Proof. Since for $F=f=0$, Lemma 3.2 proves that both $v$ and $-v$ are nonpositive, we have the uniqueness. But this implies the existence by Cramer's rule.

We now introduce the discrete Green's function $G_{h}(x, y)$ defined for each fixed $y \in \bar{R}_{h}$ by

$$
\begin{aligned}
L_{h} G_{h}(x, y) & =h^{-N} \delta(x, y) \quad x \in R_{h}, \\
G_{h}(x, y) & =\delta(x, y) \quad x \in \partial R_{h},
\end{aligned}
$$

where $\delta(x, x)=1, \delta(x, y)=0$ for $x \neq y$. In terms of this function we have the following representation:

Lemma 3.4. Let $v \in \bar{D}_{h}$. Then for $x \in \bar{R}_{h}$ we have

$$
v(x)=h^{N} \sum_{y \in R_{h}} G_{h}(x, y) L_{h} v(y)+\sum_{y \in \partial R_{h}} G_{h}(x, y) v(y) .
$$

Proof. This follows immediately from the definition of $G_{h}$ and the uniqueness part of Lemma 3.3.

We collect some simple properties of $G_{h}$ in a lemma:

Lemma 3.5. The Green's function defined above satisfies

$$
\begin{array}{rlrl}
G_{h}(x, y) & \geqq 0, & & x, y \in \bar{R}_{h} \\
\sum_{y \in \partial R_{h}} G_{h}(x, y) & \leqq 1, & x \in \bar{R}_{h}
\end{array}
$$

and there are positive constants $h_{0}$ and $C$ such that for $h \leqq h_{0}$,

$$
h^{N} \sum_{y \in R_{h}} G_{h}(x, y) \leqq C, \quad x \in \bar{R}_{h} .
$$

Proof. The nonnegativity follows at once from the definition and Lemma 3.2, and (3.5) then follows by setting $v \equiv 1$ in (3.4) and noticing that by (2.3), $L_{h} 1 \geqq 0$. Because of the assumptions on $L$ there exists a function $\phi \in \mathfrak{e}^{2}(\bar{\Omega})$ satisfying $L \phi \geqq 2$, and by consistency for sufficiently small $h$ and $x \in R_{h}$, we have $L_{h} \phi(x) \geqq 1$. Setting $v=\phi$ in (3.4) and using (3.5) we therefore obtain (3.6).

In order to give the next lemma which is the crucial lemma for our theorem,we shall need some further notation. Let $d(x)$ denote as before the distance from any point $x \in R$ to $\partial R$. Since we have assumed $\partial R \in \mathbb{C}^{\infty}$ then if $2 \delta_{0}>0$ is less than the 
minimum over $\partial R$ of the radius of the osculating sphere, we also have $\partial R_{\delta} \in \mathcal{C}^{\infty}$ for $\delta<2 \delta_{0}$ and $d \in \mathcal{C}^{\infty}\left(\bar{\Re} \backslash \mathbb{R}_{2 \delta_{0}}\right)$. For any nonnegative integer $j$ we define

$$
P_{h, j}=\left\{x ; x \in R_{h}, \frac{1}{2} \mathfrak{K} j h<d(x) \leqq \frac{1}{2} \mathfrak{K}(j+1) h\right\},
$$

where $\mathfrak{K}$ is the constant in Lemma 3.1 .

We shall then have the following (this is the first time any regularity of $\partial R$ need be assumed):

Lemma 3.6. There are positive constants $C$ and $h_{0}$ such that when $h \leqq h_{0}, \frac{1}{2} \pi j h<$ $\delta_{0}$, we have

$$
h^{N-1} \sum_{y \in P_{h, j}} G_{h}(x, y) \leqq C j h .
$$

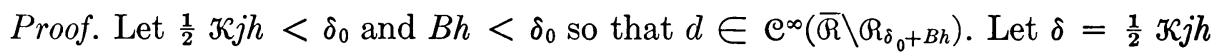
and set

$$
\begin{aligned}
\phi_{\delta}(x) & =\delta \quad x \in R_{\delta} \\
& =d(x) \quad x \in \bar{R} \backslash \Re_{\delta} .
\end{aligned}
$$

We want to apply Lemma 3.4 to the restriction of $\phi_{\delta}$ to $\bar{R}_{h}$. We have

$$
\begin{aligned}
L_{h} \phi_{\delta}(y) & =L_{h} d(y)+h^{-2} \sum_{y+\beta h \in R_{h} \cap \Re_{\delta}} b_{\beta}(y, h)[\delta-d(y+\beta h)] \\
& \geqq L_{h} d(y), \quad y \in R_{h} \backslash \Re_{\delta}
\end{aligned}
$$

and since $L_{h} \delta \geqq 0$ by $(2.3)$

$$
\begin{array}{r}
L_{h} \phi_{\delta}(y)=L_{h} \delta+h^{-2} \sum_{y+\beta h \notin R_{h} \backslash \Re_{\delta}} b_{\beta}(y, h)[d(y+\beta h)-\delta] \geqq 0, \\
y \in R_{h} \cap \Re_{\delta} .
\end{array}
$$

We need a stronger result for $y \in P_{h, j}$. To this end let $\eta$ be the exterior normal at $y$ of $\partial R_{d(y)}$ and notice that the distance from $y$ to $\partial R$ is attained in the direction of $\eta$. It follows from Lemma 3.1 that there is a $\beta$ such that

$$
(\beta h, \eta) \geqq \Re h, \quad-b_{\beta}(y, h) \geqq \Re
$$

and since the distance from $y$ to $\partial \mathscr{R}_{\delta}$ is at most $\frac{1}{2} \mathcal{K} h$ we can conclude that for some positive $h_{0}$ depending on the curvature of $\partial R$ and on $B$, we have for $h \leqq h_{0}$ that $d(y+\beta h) \leqq \delta-\frac{1}{4} \mathcal{K} h$ and it follows that

$$
L_{h} \phi_{\delta}(y) \geqq C h^{-1}, \quad y \in P_{h, j} .
$$

Using Lemma 3.4 with $v=\phi_{\delta}$, we now see from (3.7), (3.8), and (3.9) that

$$
h^{N-1} \sum_{y \in P_{h}, j} G_{h}(x, y) \leqq C\left\{\phi_{\delta}(x)+h^{N} \sum_{l<j} \sum_{y} \in_{P h, l} G_{h}(x, y)\left|L_{h} d(y)\right|\right\}
$$

and using the definition of $\phi_{\delta}$ and that fact that $d \in \mathcal{C}^{\infty}\left(\bar{\Omega} \backslash R_{2 \delta_{0}}\right)$ we have for $\frac{1}{2} \mathfrak{K} j h<\delta_{0}$,

$$
h^{N-1} \sum_{y \in P_{h, j}} G_{h}(x, y) \leqq C\left\{\delta+h^{N} \sum_{l<j} \sum_{y \in P_{h}, l} G_{h}(x, y)\right\} .
$$

Since by (3.6) the quantity on the right is bounded independently of $j$, we get by summation over $j$ and multiplication by $h$, 


$$
h^{N} \sum_{l<j} \sum_{y \in P_{h}, l} G_{h}(x, y) \leqq C \delta
$$

which together with (3.10) proves the result.

4. Some Estimates for the Continuous Problem. We start by quoting some definitions and results on interpolation spaces. For generalities, see [10] and references.

Let $B_{j}, j=0,1$, be two Banach spaces with $B_{1} \subseteq B_{0}$ so that for the corresponding norms,

$$
\|u\|_{B_{0}} \leqq C\|u\|_{B_{1}} .
$$

We set for $t>0$,

$$
K(t, u)=\inf _{v \in B_{1}}\left(\|u-v\|_{B_{0}}+t\|v\|_{B_{1}}\right)
$$

and denote for $0<\theta<1$ by $\left(B_{0}, B_{1}\right)_{\theta}$ the subspace of $B_{0}$ defined by

$$
\|u\|_{\left(B_{0}, B_{1}\right) \theta}=\sup _{t>0} t^{-\theta} K(t, u)<\infty \text {. }
$$

We have $B_{1} \subseteq\left(B_{0}, B_{1}\right)_{\theta} \subseteq B_{0}$, and for $B_{1}=B_{0}$,

$$
\|u\|_{\left(B_{0}, B_{0}\right) \theta}=\|u\|_{B_{0}} .
$$

We first state the following interpolation property:

Lemma 4.1. Let $B_{j}, B{ }^{\prime}, j=0,1$, for four Banach spaces with $B_{1} \subseteq B_{0}, B_{1}{ }^{\prime} \subseteq B_{0}{ }^{\prime}$, and let $A$ be a linear operator from $B_{0}$ into $B_{0}{ }^{\prime}$ such that for $u \in B_{1}, A u \in B_{1}{ }^{\prime}$, and

$$
\|A u\|_{B_{j}{ }^{\prime}} \leqq C_{j}\|u\|_{B_{j}}, \quad j=0,1 .
$$

Then for $u \in\left(B_{0}, B_{1}\right)_{\theta}=B_{\theta}$ we have $A u \in\left(B_{0}{ }^{\prime}, B_{1}{ }^{\prime}\right)_{\theta}=B_{\theta^{\prime}}$ and

$$
\|A u\|_{B_{\theta}{ }^{\prime}} \leqq C_{0}{ }^{1-\theta} C_{1}{ }^{\theta}\|u\|_{B \theta}, \quad 0<\theta<1 .
$$

In our applications, the Banach spaces will be of the type $\mathfrak{C}(\bar{\Omega}), \mathfrak{C}^{\lambda}\left(\bar{\Omega}_{\delta}\right), \mathfrak{C}^{\mu}(\partial \Re)$, etc. We shall need the following facts:

LEMma 4.2. With the above notation we have for $0 \leqq p_{0} \leqq p_{1}$ and with $p=p_{0}+$ $\theta\left(p_{1}-p_{0}\right)$

$$
\begin{aligned}
\left(\mathfrak{C}^{p_{0}}(\bar{\Re}), \mathfrak{e}^{p_{1}}(\bar{R})\right)_{\theta}=\mathfrak{C}_{Z}^{p}(\bar{R}) \\
\left(\mathfrak{C}^{p_{0}}(\partial R), \mathfrak{C}^{p_{1}}(\partial R)\right)_{\theta}=\mathfrak{C}_{Z}^{p}(\partial R)
\end{aligned}
$$

where equality signifies equivalence of the respective norms.

We shall now collect some well-known inequalities for the Dirichlet problem (2.1), (2.2). For proofs, see e.g. Miranda [9]. We shall always assume that $L$ and $R$ satisfy the conditions in Section 2 , in particular that $a_{0} \geqq 0$ in $R$. First we have the maximum principle estimate:

Lemma 4.3. There is a constant $C$ such that for $u \in \mathbb{e}^{2}(R) \cap \mathfrak{C}(\bar{R})$ we have

$$
|u|_{R} \leqq C\left\{|L u|_{R}+|\tilde{u}|_{\partial \Re}\right\} \text {. }
$$

The following two lemmas contain the interior and up-to-the-boundary Schauder estimates. 
Lemma 4.4. If $\lambda$ is a positive noninteger, there is a constant $C$ such that for $u \in$ $\mathfrak{C}^{2+\lambda}(R) \cap \mathfrak{e}(\bar{\Re})$

$$
|u|_{2+\lambda, R \delta} \leqq C \delta^{-2-\lambda}\left\{|L u|_{\lambda, \mathbb{R}}+|u|_{\mathbb{R}}\right\} .
$$

Lemma 4.5. If $\lambda$ is a positive noninteger, there is a constant $C$ such that for $u \in$ $\mathfrak{C}^{2+\lambda}(\bar{\Omega})$,

$$
|u|_{2+\lambda, R} \leqq C\left\{|L u|_{\lambda, R}+|\tilde{u}|_{2+\lambda, \partial R}\right\} .
$$

For a general uniformly elliptic operator there would have been a term $|u|_{\Omega}$ on the right in this inequality, but here this term can be estimated by Lemma 4.3.

Lemmas 4.3 through 4.5 can be used to prove the following existence and uniqueness result:

Lemma 4.6. Let $\lambda$ be a positive noninteger. Then if $F \in \mathcal{C}^{\lambda}(\bar{\Omega}), f \in \mathfrak{C}(\partial \Re)$ the Dirichlet problem (2.1), (2.2) has a unique solution $u \in \mathfrak{C}^{2+\lambda}(\Re) \cap \mathfrak{e}(\bar{\Omega})$. If in addition $f \in \mathrm{e}^{2+\lambda}(\partial \Re)$ we have $u \in \mathfrak{C}^{2+\lambda}(\bar{\Re})$.

Using the above interpolation lemma we shall now derive some auxiliary inequalities for the Dirichlet problem

$$
\begin{aligned}
L u=0 & \text { in } R, \\
u=f & \text { on } \partial R .
\end{aligned}
$$

Lemma 4.7. Let $\lambda$ be a positive noninteger and $0 \leqq \mu \leqq 2+\lambda$. Then there is a constant $C$ such that for $f \in \mathcal{C}_{Z}{ }^{\mu}(\partial R)$ the solution $u$ of (4.1), (4.2) satisfies

$$
|u|_{2+\lambda, \Re \delta} \leqq C \delta^{\mu-2-\lambda}|f|_{Z, \mu, \partial \Omega} .
$$

Proof. We have by Lemmas 4.3 and 4.4

$$
|u|_{2+\lambda, Q \delta} \leqq C \delta^{-2-\lambda}|f|_{\partial R}
$$

and by Lemma 4.5,

$$
|u|_{2+\lambda, \Re \delta} \leqq|u|_{2+\lambda, \Re} \leqq C|f|_{2+\lambda, \partial \Omega} .
$$

The result therefore follows by applying Lemmas 4.1 and 4.2 to the operator which takes $f$ into the solution $u \in \mathbb{C}^{2+\lambda}\left(\overline{\mathfrak{R}}_{\delta}\right)$ of (4.1), (4.2).

Lemma 4.8. If $\mu$ is positive there is a constant $C$ such that for $f \in \mathfrak{C}_{Z}{ }^{\mu}(\partial \Re)$ the solution $u$ of (4.1), (4.2) belongs to $\mathfrak{C}_{Z}{ }^{\mu}(\bar{\Omega})$ and satisfies

$$
|u|_{Z, \mu, \Omega} \leqq C|f|_{Z, \mu, \partial \Omega} .
$$

Proof. For $2+\lambda>\mu$ and nonintegral we have again

$$
|u|_{2+\lambda, \Re} \leqq C|f|_{2+\lambda, \partial R}
$$

and by Lemma 4.3

$$
|u|_{R} \leqq C|f|_{\partial R} .
$$

The result therefore follows by Lemmas 4.1 and 4.2 .

5. The Rate of Convergence. In this section we shall establish the unique solvability of the discrete Dirichlet problem (2.5), (2.6) and discuss the rate of convergence of its solution $u_{h}$ to the solution $u$ of the continuous problem (2.1), 
(2.2). More precisely we shall prove a sequence of lemmas leading up to the proof of our Theorem as stated in Section 2. Throughout this section we shall assume that the operators $L, L_{h}, l_{h}, M_{h}$, and $m_{h}$ satisfy the assumptions of Section 2 .

We first have the following two simple estimates:

Lemma 5.1. For any mesh-function $u \in \overline{\mathfrak{D}}_{h}$ we have

$$
|u|_{\partial R_{h}} \leqq \gamma|u|_{\bar{R}_{h}}+\left|l_{h} u\right|_{\partial R_{h}}
$$

where $\gamma<1$ is the constant in (2.4).

Proof. This is an immediate consequence of the definition of the operator $l_{h}$.

Lemma 5.2. There are positive constants $h_{0}$ and $C$ such that for $h \leqq h_{0}$ and $u \in \mathbb{D}_{h}$ we have

$$
|u|_{\bar{R}_{h}} \leqq C\left\{\left|L_{h} u\right|_{R_{h}}+\left|l_{h} u\right|_{\partial R_{h}}\right\} .
$$

Proof. We have by Lemmas 3.4 and 3.5

$$
\begin{aligned}
|u(x)| & \leqq h^{N} \sum_{y \in R_{h}} G_{h}(x, y)\left|L_{h} u(y)\right|+\sum_{y \in \partial R_{h}} G_{h}(x, y)|u(y)| \\
& \leqq C\left|L_{h} u\right|_{R_{h}}+|u|_{\partial R_{h}},
\end{aligned}
$$

and the result therefore follows from Lemma 5.1.

As a consequence we can now prove the existence of a solution of the discrete problem.

Lemma 5.3. With the $h_{0}$ in Lemma 5.2, the discrete problem (2.5), (2.6) has a unique solution $u_{h}$ for $h \leqq h_{0}$ and arbitrary choice of $F$ and $f$.

Proof. Uniqueness is an immediate consequence of Lemma 5.2 and as in Lemma 3.3 , uniqueness implies existence.

We can now essentially prove the convergence result in the case of homogeneous boundary conditions:

Lemma 5.4. Assume that the discrete problem (2.5), (2.6) approximates the continuous problem (2.1), (2.2) with order of accuracy $\nu$ and let $\lambda \geqq 0, \lambda \neq \nu$. Then there is a constant $C$ such that if $F \in \mathcal{C}_{z}{ }^{\lambda}(\bar{R})$ and if $u$ and $u_{h}$ are the solutions of (2.1), (2.2) and (2.5), (2.6), respectively, with $f=0$, then

$$
\left|u-u_{h}\right|_{R_{h}} \leqq C h^{\min (\lambda, \nu)}|F|_{Z, \lambda, R} .
$$

Proof. Since $M_{h}$ and $m_{h}$ are bounded we obtain by Lemmas 4.3 and 5.2

$$
\left|u-u_{h}\right|_{R_{h}} \leqq|u|_{\Re}+\left|u_{h}\right|_{R_{h}} \leqq C|F|_{\Re}
$$

which is (5.3) in the case $\lambda=0$. For $\lambda>0$, it is clearly, by Lemmas 4.1 and 4.2, no restriction of the generality to assume that $\lambda$ is a noninteger. We then have $u \in$ $\mathfrak{C}^{2+\lambda}(\bar{R})$ by Lemma 4.6 . We want to apply Lemma 5.2 to $u-u_{h}$. We have by $(2.7)$ and Lemma 4.5,

$$
\begin{aligned}
\left|L_{h}\left(u-u_{h}\right)\right|_{R_{h}} & =\left|L_{h} u-M_{h} L u\right|_{R_{h}} \\
& \leqq C h^{\min (\lambda, \nu)}|u|_{2+\lambda, \Omega} \leqq C h^{\min (\lambda, \nu)}|F|_{\lambda, Q}
\end{aligned}
$$

and similarly by (2.8),

$$
\begin{aligned}
\left|l_{h}\left(u-u_{h}\right)\right|_{\partial R_{h}} & =\left|l_{h} u-m_{h}(L u, 0)\right|_{\partial \Omega_{h}} \\
& \leqq C h^{\min (\lambda, \nu)}\left(|u|_{\lambda, \Omega}+|L u|_{\Omega}\right) \leqq C h^{\min (\lambda, \nu)}|F|_{\lambda, \Omega} .
\end{aligned}
$$


Together (5.1), (5.4) and (5.5) prove the lemma.

For the treatment of the homogeneous equation we need an a priori inequality which is somewhat stronger than (5.1). For this purpose we introduce the norm

$$
\|u\|_{R_{h}}=h^{2} \sum_{\varkappa_{j h \leq 2 \delta_{0}}} j|u|_{P_{h, j}}+|u|_{R_{\delta_{0}, h}}
$$

where $\delta_{0}$ is the positive number introduced in Section 3 and $R_{\delta_{0}, h}=\Re_{\delta_{0}} \cap R_{h}$. We clearly have for some $C$ independent of $h$,

$$
\|u\|_{R_{h}} \leqq C|u|_{R_{h}}
$$

but the new norm gives less weight to the values of $u$ near $\partial R_{h}$; it can be thought of as a discrete analogue of

$$
\|u\|_{\Omega}=\int_{0}^{\delta_{0}} \delta|u| \partial \Re_{\delta} \partial \delta+|u| \Re_{\delta_{0}} .
$$

With this norm, we then have

Lemma 5.5. There are positive constants $h_{0}$ and $C$ such that for $h \leqq h_{0}$ and $u \in \bar{D}_{h}$ we have

$$
|u|_{\bar{R}_{h}} \leqq C\left\{\left\|L_{h} u\right\|_{R_{h}}+\left|l_{h} u\right|_{\partial R_{h}}\right\} .
$$

Proof. We have by Lemmas 3.5 and 3.6,

$$
\begin{aligned}
h^{N} \sum_{y \in R_{h}} G_{h}(x, y)\left|L_{h} u(y)\right| \leqq & h \sum_{\varkappa_{j h \leqq 2 \delta_{0}}}\left|L_{h} u\right|_{P_{h, j}} h^{N-1} \sum_{y \in P_{h, j}} G_{h}(x, y) \\
& +\left|L_{h} u\right|_{R_{\delta_{0}, h}} h^{N} \sum_{y \in R_{h}} G_{h}(x, y) \leqq C\left\|L_{h} u\right\|_{R_{h}} .
\end{aligned}
$$

The result therefore follows as above from (5.2).

We can now prove the following convergence result for the homogeneous equation:

Lemma 5.6. Assume that the discrete problem (2.5), (2.6) approximates the continuous problem (2.1), (2.2) with order of accuracy $\nu$ and let $\mu \geqq 0, \mu \neq \nu$. Then there is a constant $C$ such that if $f \in \mathcal{C}_{Z}{ }^{\mu}(\partial R)$ and if $u$ and $u_{h}$ are the solutions of (2.1), (2.2) and (2.5), (2.6) respectively, with $F=0$, then

$$
\left|u-u_{h}\right|_{R_{h}} \leqq C h^{\min (\mu, \nu)}|f|_{z, \mu, \partial \Omega} .
$$

Proof. As in the proof of Lemma 5.4, we first notice that by Lemmas 4.3 and 5.2, (5.6) holds for $\mu=0$, and that we can then assume without loss of generality that $\mu$ is a noninteger. By Lemma 4.8 we have $u \in \mathcal{C}^{\infty}(\Re) \cap \mathfrak{e}^{\mu}(\bar{\Omega})$. We want to apply Lemma 5.5 to $u-u_{h}$. We have

$$
\begin{aligned}
\| L_{h}\left(u-u_{h}\right)_{\| R_{h}} & =\left\|L_{h} u\right\|_{R_{h}} \\
& \leqq C\left\{h^{2} \sum_{y, 4 B h \leqq \mathcal{K} j h \leqq 2 \delta_{0}} j\left|L_{h} u\right|_{P_{h, j}}+h^{2}\left|L_{h} u\right|_{R_{h}}+\left|L_{h} u\right|_{R_{\delta_{0}, h}}\right\} .
\end{aligned}
$$

Consider the second term. By the definition of $L_{h}$ and Lemma 4.3 it follows that

$$
h^{2}\left|L_{h} u\right|_{R_{h}} \leqq C|f|_{\partial \Omega} .
$$

Also for any positive noninteger $\bar{\nu}$ such that $\nu<\bar{\nu}+2<\nu+2$ we have from (2.7) and Lemma 4.8 that 


$$
h^{2}\left|L_{h} u\right|_{R_{h}} \leqq C h^{2+\bar{\nu}}|f|_{2+\bar{\nu}, \partial \Omega} .
$$

We may now apply Lemma 4.1 to the operator which takes $f$ into $h^{2} L_{h} u \in D_{h}$ (with maximum norm) to obtain

$$
h^{2}\left|L_{h} u\right|_{R_{h}} \leqq C h^{\mu}|f|_{Z, \mu, \partial \Omega}, \quad 0 \leqq \mu \leqq 2+\bar{\nu} .
$$

Clearly this implies that

$$
h^{2}\left|L_{h} u\right|_{R_{h}} \leqq C h^{\min (\mu, \nu)}|f|_{\mu, \partial \Omega}
$$

for all $\mu \geqq 0$.

The last term can be estimated by applying (2.7) and the proof of Lemma 4.7:

$$
\left|L_{h} u\right|_{R_{\delta_{0}, h}} \leqq C h^{\nu}|u|_{2+\nu,\left(\delta_{0} / 2\right.} \leqq C h^{\nu}|f|_{\partial \Omega} \leqq C h^{\min (\mu, \nu)}|f|_{\mu, \partial \Omega} .
$$

Consider now the sum on the right in (5.7). For $y \in P_{h, j}$ with $4 B h \leqq \mathcal{K} j h$ we have

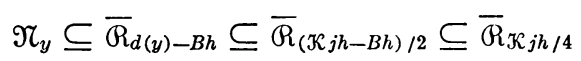

and thus by (2.7) and Lemma 4.7

$$
\left|L_{h} u\right|_{P_{h, j}} \leqq C h^{\min (\lambda, \nu)}|u|_{2+\lambda, Q \Re j h / 4} \leqq C h^{\min (\lambda, \nu)}(j h)^{\mu-2-\lambda}|f|_{\mu, \partial \Omega} .
$$

We obtain

$$
h^{2} \sum_{4 B h \leqq \mathcal{K} j h \leqq 2 \delta_{0}} j\left|L_{h} u\right|_{P h, j} \leqq C h^{\min (\lambda, \nu)} \sum_{4 B h \leqq \mathcal{K} j h \leqq 2 \delta_{0}}(j h)^{-1+\mu-\lambda} h|f|_{\mu, \partial \Omega} .
$$

Since

$$
\begin{aligned}
\sum_{4 B h \leqq \mathcal{K} j h \leqq 2 \delta_{0}}(j h)^{-1+\mu-\lambda} h & \leqq C \text { if } \mu>\lambda, \\
& \leqq C h^{\mu-\lambda} \text { if } \mu<\lambda
\end{aligned}
$$

we can now choose $\lambda$ between $\mu$ and $\nu$ and obtain by (5.10)

$$
h^{2} \sum_{4 B h \leqq \mathcal{K} j h \leqq 2 \delta_{0}} j\left|L_{h} u\right|_{P_{h, j}} \leqq C h^{\min (\mu, \nu)}|f|_{\mu, \delta \Omega} .
$$

Together (5.7), (5.8), (5.9) and (5.11) prove

$$
\left\|L_{h}\left(u-u_{h}\right)\right\|_{R_{h}} \leqq C h^{\min (\mu, \nu)}|f|_{\mu, \partial \Omega} .
$$

Finally

$$
\begin{aligned}
\left|l_{h}\left(u-u_{h}\right)\right|_{\partial R_{h}} & =\left|l_{h} u-m_{h}(0, \tilde{u})\right|_{\partial R_{h}} \leqq C h^{\min (\mu, \nu)}|u|_{\mu, \Re} \\
& \leqq C h^{\min (\mu, \nu)}|f|_{\mu, \partial \Re}
\end{aligned}
$$

and by Lemma 5.5, (5.12) and (5.13) prove the lemma.

We can now complete the proof of the theorem. Let first $\lambda, \mu \geqq 0, \lambda, \mu \neq \nu$, and let $u_{h}^{(j)}$ and $u^{(j)}, j=1,2$, be the solutions of the discrete and continuous problems corresponding to $\mathcal{F}=(F, 0)$ and $\mathcal{F}^{\cdot}=(0, f)$, respectively. We then obviously have $u=u^{(1)}+u^{(2)}$ and $u_{h}=u_{h}^{(1)}+u_{h}^{(2)}$ and by Lemmas 5.4 and 5.6 we therefore get

$$
\begin{aligned}
\left|u-u_{h}\right|_{\bar{R}_{h}} & \leqq\left|u^{(1)}-u_{h}^{(1)}\right|_{\bar{R}_{h}}+\left|u^{(2)}-u_{h}{ }^{(2)}\right|_{\bar{R}_{h}} \\
& \leqq C\left\{h^{\min (\lambda, \nu)}|F|_{Z, \lambda, \mathcal{R}}+h^{\min (\mu, \nu)}|f|_{Z, \mu, \partial \mathcal{G}}\right\}
\end{aligned}
$$

which is (2.9). 
Assume now that $F \in \mathcal{C}^{\lambda}(\bar{R})$ and $f \in \mathcal{C}(\partial R)$. Given $\epsilon>0$ we can find $\tilde{f} \in$ $\mathfrak{C}^{\lambda}(\partial \Re)$ such that $|f-\tilde{f}|_{\partial \Omega}<\epsilon$. Let $\tilde{F}=(F, \tilde{f})$ and let $\tilde{u}_{h}$ and $\tilde{u}$ be the solutions of the corresponding discrete and continuous problems, respectively. We then have

$$
\left|u-u_{h}\right|_{\bar{R}_{h}} \leqq|u-\tilde{u}|_{\Omega}+\left|u_{h}-\tilde{u}_{h}\right|_{\bar{R}_{h}}+\left|\tilde{u}-\tilde{u}_{h}\right|_{\bar{R}_{h}} .
$$

By Lemmas 4.3 and 5.2, and since $M_{h}$ and $m_{h}$ are bounded and linear we have

$$
|u-\tilde{u}|_{R}+\left|u_{h}-\tilde{u}_{h}\right|_{\bar{R}_{h}} \leqq C|f-\tilde{f}|_{\partial \Omega} \leqq C \epsilon .
$$

Since $\tilde{F} \in \mathcal{C}^{\lambda}(\bar{R}) \times \mathfrak{C}^{\lambda}(\partial R)$, we have

$$
\lim _{h \rightarrow 0}\left|\tilde{u}-\tilde{u}_{h}\right|_{\bar{R}_{h}}=0
$$

by (5.14), and the result therefore follows from (5.15) and (5.16).

Remark. The interpolation technique can also be used to simplify the definition (2.7), (2.8) of the order of accuracy. Assume e.g. that (2.7) holds with $\lambda=\nu$ and that in addition the operator $M_{h}$ has the property that for some $C$,

$$
\left|M_{h} F(x)\right| \leqq C|F|_{\Re_{x}}, \quad x \in R .
$$

By consistency we find

$$
\left|L_{h} u(x)\right| \leqq C|u|_{2, \Re_{x}}
$$

and therefore, (2.7) holds also with $\lambda=0$. Hence, (2.7) holds for general $\lambda$ with $0 \leqq \lambda \leqq \nu$ by Lemmas 4.1 and 4.2 (with $\Re_{x}$ instead of $R$ ).

\author{
Cornell University \\ Mathematics Department \\ Ithaca, N. Y. 14850 \\ University of Maryland \\ Institute for Fluid Dynamics and Applied Mathematics \\ College Park, Maryland 20740
}

Chalmers Institute of Technology

Göteborg, Sweden

1. N. S. Bahvalov, "Numerical solution of the Dirichlet problem for Laplace's equation," Vestnik Moskov. Univ. Ser. Mat. Meh. Astronom. Fiz. Him., v. 3, 1959, no. 5, pp. 171-195. (Russian) MR $22 \# 6082$

2. J. H. Bramble, "On the convergence of difference approximations for second order uniformly elliptic operators" in Numerical Solution of Field Problems in Continuum Physics, SIAMAMS Proceedings, vol. 2, Amer. Math. Soc., Providence, R. I. (To appear.)

3. J. H. Bramble \& B. E. Hubbard, "On the formulation of finite difference analogues of the Dirichlet problem for Poisson's equation," Numer. Math., v. 4, 1962, pp. 313-327. MR 26 \#7157.

4. J. H. BRAmble \& B. E. HuBbard, "A theorem on error estimation for finite difference analogues of the Dirichlet problem for elliptic equations," Contributions to Differential Equations, v. 2, 1963, pp. 319-340. MR 27 \#2114.

5. J. H. Bramble, R. B. KellogG \& V. Thomée, "On the rate of convergence of some difference schemes for second order elliptic equations," Nordisk Tidskr. Informations-Behandling, v. 8,1968 , pp. $154-173$.

6. L. Collatz, "Bemerkungen zur Fehlerabschätzung für das Differenzenverfahren bei partiellen Differentialgleichungen," $Z$. Angew. Math. Mech., v. 13, 1933, pp. 56-57.

7. S. Gerschgorin, "Fehlerabschätzung für das Differenzenverfahren zur Lösung partieller Differentialgleichungen," Z. Angew. Math. Mech., v. 10, 1930, pp. 373-382.

8. P. LaAsonen, "On the solution of Poisson's difference equation," J. Assoc. Comput. Mach., v. 5, 1958, pp. 370-382. MR $22 \# 12726$. 
9. C. Miranda, Equazioni alle Derivate Parziali di Tipo Ellittico, Ergebnisse der Mathematik und ihrer Grenzgebiete, Heft 2, Springer-Verlag, Berlin, 1955.

10. J. Peetre \& V. ThomÉe, "On the rate of convergence for discrete initial-value problems," Math. Scand., v. 21, 1967, pp. 159-176.

11. E. A. Volkov, "Obtaining an error estimate for a numerical solution of the Dirichlet problem in terms of known quantities," Ž. Vyčisl. Mat. i Mat. Fiz., v. 6, 1966, no. 4, suppl., pp. 5-17. (Russian) MR 35 \#2503.

12. E. A. VolKov, "Effective estimates of the error in solutions by the method of nets of boundary for the Laplace and Poisson equations on a rectangle and on certain triangles," Trudy Mat. Inst. Steklov., v. 74, 1966, pp. 55-85 = Proc. Steklov Inst. Math., v. 74, 1966, pp. 57-90. MR 37 \#1098.

13. W. WASOW, "On the truncation error in the solution of Laplace's equation by finite differences," J. Res. Nat. Bur. Standards, v. 48, 1952, pp. 345-348. MR 14, 93. 